\title{
SUMMARY 4
}

\section{Pharmacotherapy}

Serotonergic modulation of visceral sensation: upper gut Selective serotonin reuptake inhibitors (SSRIs), and 5- $\mathrm{HT}_{4}, 5-\mathrm{HT}_{1 \mathrm{p}}$, and $5-\mathrm{HT}_{1 \mathrm{~A}}$ receptor agonists all have an effect on postprandial tone in the stomach and therefore have therapeutic potential for the treatment of visceral hypersensitivity. Further clinical evaluation of these agents should focus on collecting data at the time of peak postprandial symptoms, which occurs approximately 75 minutes after a meal, and data collection should continue for up to four hours.

\section{Serotonergic modulation of visceral sensation: lower gut} Novel serotonergic agents have a significant impact on symptoms of irritable bowel syndrome (IBS) as a result of their visceral analgesic properties and diverse effects on motor function in the lower gut. Antagonism of 5-HT receptors in the sensory apparatus reduces visceral pain whereas $5-\mathrm{HT}_{3}$ inhibition in the motor apparatus retards colonic transit and enhances small intestinal absorption. Alosetron, a selective 5-HT, antagonist, has been shown to relieve pain, normalise bowel frequency, and reduce urgency in female patients with diarrhoea predominant IBS. Alosetron was withdrawn because of reports of significant adverse events, including ischaemic colitis and constipation that led to hospitalisations and surgery.

$5-\mathrm{HT}_{4}$ receptors elicit the depolarising actions of serotonin that result in the release of neurotransmitters, such as acetylcholine, from enteric neurones, while in the CNS they modulate dopamine release and have a direct role in cognition and memory. The partial 5- $\mathrm{HT}_{4}$ agonist tegaserod activates gastrointestinal motility by binding to enteric cholinergic neurones. Tegaserod has been shown to ameliorate symptoms in female patients with constipation predominant IBS, and the full $5-\mathrm{HT}_{4}$ agonist prucalopride has been shown to increase the number of spontaneous and complete bowel movements in patients with functional constipation. Further work with prucalopride is on hold until a more thorough assessment of its intestinal carcinogenic potential has been conducted in experimental animals. Citalopram reduces colonic sensation to volume distension in healthy volunteers and merits further evaluation.

A common feature of the IBS studies reviewed is that of the placebo effect. One explanation for this is the chronic recurrent symptoms of IBS. Another reason is the fact that patients were asked to contact the clinic on a daily basis to report symptom severity. Thus the observed placebo response was probably a composite of a true initial placebo effect coupled with natural symptom regression. These observations suggest that 12 week trials in IBS are less likely to be meaningful than short term trials of 4-6 weeks' duration, and in view of the cyclical nature of the condition the time to restoration of normality may be the most appropriate clinical outcome.

\section{Non-serotonergic modulation of sensation}

Pathways other than those modulated by serotonin may also be involved in the perception of visceral sensation. The additional targets for therapeutic activity include opioid, somatostatin, $\alpha_{2}$ adrenoceptors, and noradrenaline reuptake sites.

Acute inflammation produces hyperalgesia, and if the inflammation persists, locally acting opioids are produced. This longer term response is sometimes accompanied by activation of central descending nociceptive inhibitory pathways. It is possible therefore that an endogenous opioid system is deficient in patients with IBS. If this were so then giving opioids would be expected to provide clinical benefit. In accordance with this hypothesis, visceral sensitivity triggered by rectal distension in humans has been shown to be reduced by both centrally (fentanyl) and peripherally acting (fedotozine) opioids. Centrally acting opioids are not generally well tolerated. Loperamide, which has the fewest central effects, improves stool consistency and pain in patients with diarrhoea predominant IBS but in other patients its constipating effect is a problem. Fedotozine was developed as a non-constipating opioid but its clinical effects have so far been disappointing.

Somatostatin is found in dorsal horn neurones and in the substantia gelantinosa. It inhibits the firing of nociceptive neurones but has no effect on mechanosensitive neurones. Somatostatin has been shown to decrease perception of rectal distension in healthy volunteers and IBS patients and has a possible peripheral site of action.

The $\alpha$, adrenoceptor agonist clonidine has been shown to induce presynaptic hyperpolarisation and in this way it diminishes the release of neurotransmitters, especially acetylcholine. Clonidine increases colonic compliance in volunteers: it also delays small bowel transit (but not gastric or colonic transit) and reduces colonic tone and sensitivity to distension. Its sedative and analgesic effects suggest that it may have both central and peripheral actions.

\section{Centrally acting agents and visceral sensitivity}

Antidepressant drugs and opioid agents are often used to manage symptoms of IBS. Amitriptyline and the newer SSRIs are traditionally thought to increase pain tolerance by acting at the level of the central nervous system. Although there is no evidence to suggest that these compounds affect visceral sensitivity per se, it seem likely that peripheral effects contribute to their action.

Opiates, including morphine and newer analgesic agents such as trimebutine and fedotozine, have also been used to reduce visceral nociception. Recent data suggest that these agents have a peripheral action at the level of the dorsal root ganglia. However, it is likely that visceral stimulation induces long term alterations in the CNS, possibly mediated by centrally acting cytokines. These findings highlight the need for further research into two key areas: the relevance of peripheral serotonergic pathways in pain perception and the relevance of cytokines in the modulation of visceral hypersensitivity. 